\title{
A Search for the Resonant Absorption of Solar Axions by Atomic Nuclei
}

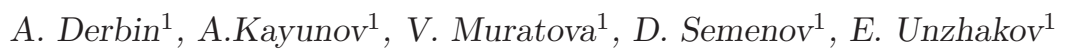 \\ ${ }^{1}$ Petersburg Nuclear Physics Institute (PNPI), Gatchina, Russia \\ DOI: http://dx.doi.org/10.3204/DESY-PROC-2011-04/unzhakov_evgeniy
}

\begin{abstract}
A search for resonant absorption of solar axions by ${ }^{57} \mathrm{Fe}$ and ${ }^{169} \mathrm{Tm}$ targets was performed. The $\mathrm{Si}(\mathrm{Li})$ detector placed inside a low-background setup was used to detect the $\gamma$-quanta appearing from the de-excitation of the low-lying nuclear levels of ${ }^{57} \mathrm{Fe}$ and ${ }^{169} \mathrm{Tm}(14.4$ $\mathrm{keV}$ and $8.41 \mathrm{keV}$ correspondingly): $A+N_{\text {target }} \rightarrow N_{\text {target }}^{*}+\gamma$. The obtained data allowed us to set the new upper limits on the axion mass value: $m_{A} \leq 145 \mathrm{eV}$ at $95 \%$ c.l.
\end{abstract}

\section{Introduction}

A natural solution of the strong CP-problem is based on the introduction of the global chiral symmetry $U(1)_{P Q}$ that was proposed by Peccei and Quinn in 1997. The spontaneous breaking of this new symmetry at the energy scale $f_{a}$ should lead to the generation of a new neutral zero-spin pseudoscalar particle - axion. Theory describes the interaction of axions with ordinary matter (nucleons, leptons and photons) in terms of effective coupling constants: $g_{A N}, g_{A e}, g_{A \gamma}$ correspondingly. The axion mass $m_{A}$ and the values of these constants appear to be inversely proportional to the $U(1)_{P Q}$ symmetry breaking scale $f_{A}$. After the first hypothesis of $f_{A} \approx 250$ $\mathrm{GeV}$ (electro-weak scale) was experimentally excluded, two types of new theoretical models were introduced. They removed the restrictions on the $f_{A}$ value, allowing it to go up to the Planck mass, therefore significantly suppressing the interaction of axions with ordinary matter and shifting the expected axion mass to the lighter region.

\section{Solar axion flux}

If axions do exist, then stars (including the Sun) should be intense sources of these particles. There are several possible mechanisms that could stand behind the production of solar axions.

Firstly, the Primakoff conversion of photons in the electromagnetic field of plasma can efficiently produce axions $(\gamma+\vec{B} \rightarrow A)$. The resulting axoin flux has an average energy of 4 $\mathrm{keV}$ and can be detected by inverse Primakoff conversion in laboratory magnetic fields or by the coherent conversion to photons in crystal detectors. These experiments are sensitive to $g_{A \gamma}$ constant. The flux of Primakoff axions is parameterized by the following expression [1]:

$$
\frac{d \Phi_{A}}{d E_{A}}=\left(g_{A \gamma}\right)^{2} \cdot 3.82 \cdot 10^{30} \frac{E_{A}^{3}}{\exp \left(E_{A} / 1.103\right)-1}
$$




\section{A SeArch for the Resonant Absorption of Solar AXions by Atomic Nuclei}

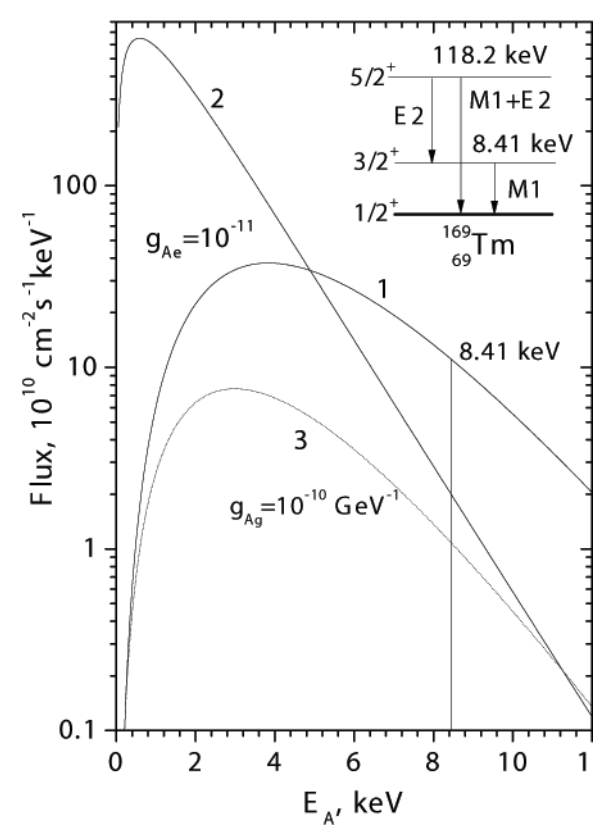

Figure 1: 1, 2 - Compton process and the bremsstrahlung axion spectra, correspondingly. 3 - Primakoff axion spectrum. The level scheme of ${ }^{169} \mathrm{Tm}$ nucleus is shown in the inset.

duced by $\mathrm{p}\left(\mathrm{d},{ }^{3} \mathrm{He}\right) \mathrm{A}$ reaction was made in [3].

Also, axions could be produced as a result of the Compton process $\left(\gamma+e^{-} \rightarrow e^{-}+A\right)$ and bremsstrahlung $\left(e^{-}+Z \rightarrow Z+e^{-}+A\right)$ in the hot solar plasma. The cross sections of these reactions depend on the axion-electron coupling constant $g_{A} e$. The expected spectrum of such axions is calculated using the theoretical predictions for the Compton cross section and the axion bremsstrahlung produced by the electron-nucleus collisions. The axion flux is determined by radial temperature distribution $T(r)$ and densities of electrons $N_{e}(r)$ and nuclei $N_{Z, A}(r)$. The resulting corresponding parameterizations of Compton and bremsstrahlung axions are [4]:

$$
\begin{gathered}
\frac{d \Phi_{A}}{d E_{A}}=g_{A e}^{2} \cdot 1.33 \cdot 10^{33} E_{A}^{2.98} e^{-0.774 E_{A}} \\
\frac{d \Phi_{A}}{d E_{A}}=g_{A e}^{2} \cdot 4.14 \cdot 10^{35} E_{A}^{0.89} e^{-0.7 E_{A}-1.26 \sqrt{E_{A}}}
\end{gathered}
$$

The total flux of solar axions produced by various processes is shown in Fig. 1. In our work we used ${ }^{169} \mathrm{Tm}$ target for resonant absorption of bremsstrahlung, Compton and Primakoff axions and ${ }^{57} \mathrm{Fe}$ for the absorption of $14.4 \mathrm{keV}$ monochromatic axions. Therefore, these experiments were sensitive to $g_{A e}, g_{A \gamma}$ and $g_{A N}$ values correspondingly. 
EVGENIY UNZHAKOV

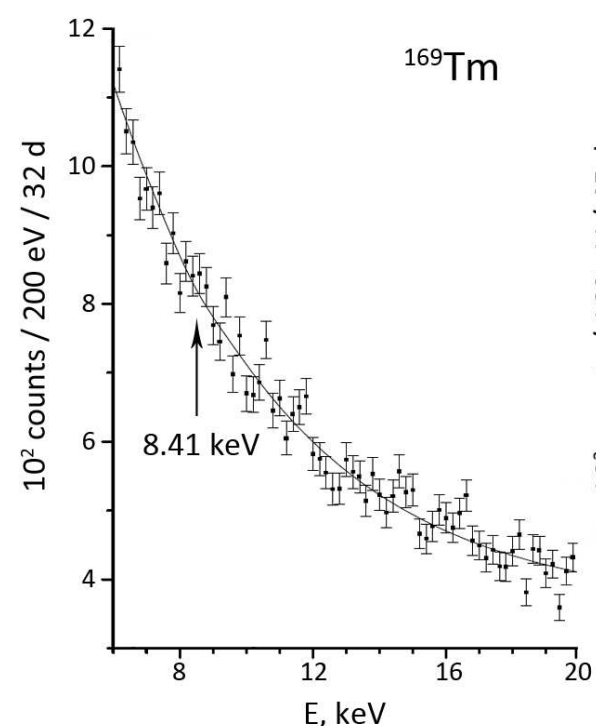

(a)

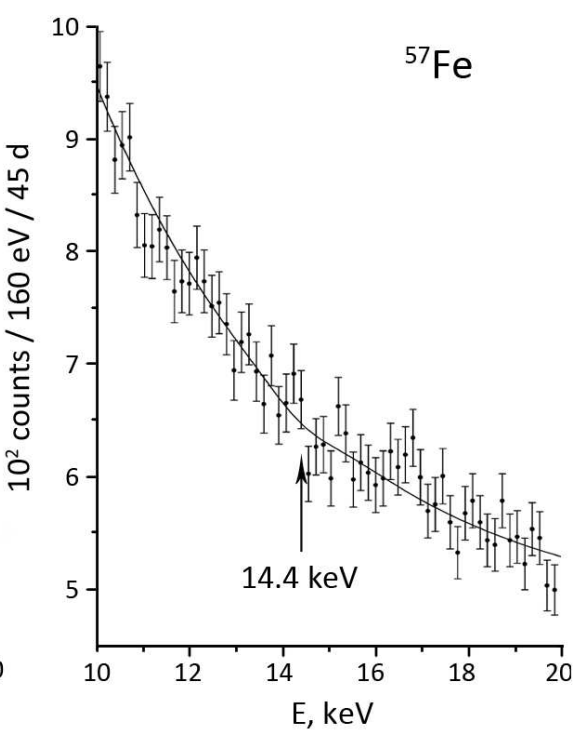

(b)

Figure 2: The $\mathrm{Si}(\mathrm{Li})$ detector energy spectra of ${ }^{169} \mathrm{Tm}$ (a) and ${ }^{57} \mathrm{Fe}(\mathbf{b})$ targets, measured in the anticoincidence with the veto signal. Solid line shows the fitting results for corresponding regions.

\section{$3 \quad$ Experimental setup}

In order to observe $8.41 \mathrm{keV}$ and $14.4 \mathrm{keV} \gamma$-rays we used the planar $\mathrm{Si}(\mathrm{Li})$ detector with a sensitive area diameter $d=66 \mathrm{~mm}$ and thickness of $5 \mathrm{~mm}$. The detector was mounted on 5 $\mathrm{cm}$ thick copper plate that protected the detector from the external radiation. The detector and the holder were placed in a vacuum cryostat and cooled to the temperature of the liquid nitrogen. The corresponding target was uniformly deposited on a Plexiglas substrate $70 \mathrm{~mm}$ in diameter at the distance of $1.5 \mathrm{~mm}$ from the detector surface. External passive shielding composed of copper, iron and lead layers was adjusted to the cryostat and suppressed the external radioactivity background by a factor of about 500 .

The experimental setup was located on the ground surface. The events produced by cosmic rays and fast neutrons were registered by the active shielding consisting of five plastic scintillators $50 \times 50 \times 12 \mathrm{~cm}$ in size. The rate of $50 \mu$ s veto signals was 600 counts $/ \mathrm{s}$ that lead to $\approx 3 \%$ of the dead time. The $\mathrm{Si}(\mathrm{Li})$ detector was sectionalized into nine separate subregions in order to reduce the capacities of individual sections and, therefore, increase the overall energy resolution. Every section was equipped with a charge-sensitive preamplifier with resistive feedback, a shaping amplifier and a 12-step ADC. Eighteen 4096-channel spectra (in coincidence and anticoincidence with the veto signal) were collected.

Though the detector amplifications were actually the same, the energy calibrations were performed for each section individually. Standard ${ }^{57} \mathrm{Co}$ and ${ }^{241} \mathrm{Am}$ calibration sources were used. The total energy resolution for a $14.4 \mathrm{keV} \gamma$-ray line was equal to $\sigma=0.63 \mathrm{keV}$. The high energy resolution and accurate knowledge of energy scale are of great importance for our experiment, because the energies of thulium characteristic x-rays are close to $8.41 \mathrm{keV}$. 


\section{A SEARch For the Resonant Absorption of Solar Axions by Atomic Nuclei}

The sensitive volume and area of the $(\mathrm{Si}) \mathrm{Li}$ detector were measured via the $\mathrm{x}$-ray and $\gamma$ lines of a standard ${ }^{241} \mathrm{Am}$ source. The detection efficiency was estimated by the MC simulation that included the self-absorption of $\gamma$-rays by the target. Total detection efficiency for the 8.41 $\gamma$-rays tured out to be $\epsilon \approx 6 \%$.

\section{Results}

The measurements were carried out during 45 days of live time with ${ }^{57} \mathrm{Fe}$ target and during 32 days of live time with ${ }^{169} \mathrm{Tm}$ target. The detailed energy spectra of the regions where axion peak was expected are presented in Fig. 2. There were no statistically significant peaks in the given regions, therefore, we used the standard method of $\chi^{2}$ profiling in order to determine the upper limits on the number of events inside the axion peak. The result of the fit corresponding to the minimum of $\chi^{2}$ is shown by a solid line in Fig. 2. The value of $\chi^{2}$ was determined for different fixed values of axion peak intensity $S_{A}$ while the other parameter were left unrestricted. The obtained probability function $P\left(\chi^{2}(S)\right)$ was normalized to unity for $S \geq 0$.

Ulitmately, the expected number of the axion peak $\gamma$-quanta would be proportional to the number of target nuclei $N_{\text {target }}$, time of measurement $T, \gamma$-ray detection efficiency $\epsilon$ and the internal converion ratio of the corresponding nuclear transition $\eta$.

The obtained results allowed us to set the following upper limits on the values of coupling constants and axion mass: $\left|g_{A N}^{0}+g_{A N}^{3}\right| \leq 3.0 \times 10^{-6}$ and $m_{A} \leq 145 \mathrm{eV}$ for ${ }^{57} \mathrm{Fe}$ meaurements; $g_{A e} \times\left|g_{A N}^{0}+g_{A N}^{3}\right| \leq 2.1 \times 10^{-14}$ and $g_{A e} \times m_{A} \leq 3.2 \times 10^{-7} \mathrm{eV}$ in case of Compton and bremsstrahlung axion absorption by ${ }^{169} \mathrm{Tm} ; g_{A \gamma} \times m_{A} \leq 1.36 \times 10^{-5}$ and $m_{A} \leq 169 \mathrm{eV}$ in case of Primakoff axion absorption by ${ }^{169} \mathrm{Tm}$. All the limits are given at $95 \%$ c.l.

For more details on the calculation of the axion absorption rates, model parameter dependencies and result comparison see [1], [2] and [4].

\section{References}

[1] A. Derbin, S. Bakhlanov, A. Egorov, I. Mitropolsky, V. Muratova, D. Semenov, E. Unzhakov, "Search for Solar Axions Produced by Primakoff Conversion Using Resonant Absorption by ${ }^{169}$ Tm Nuclei," Phys. Lett. B 678, 181-185 (2009) [arXiv:0904.3443 [hep-ex]].

[2] A. Derbin, A. Egorov, I. Mitropolsky, V. Muratova, D. Semenov, E. Unzhakov, "Search for Resonant Absorption of Solar Axions Emitted in M1 Transition in ${ }^{57}$ Fe Nuclei," Eur. Phys. J. C 62, 755-760 (2009) [arXiv:0906.0256 [hep-ex]].

[3] A. Derbin, A. Kauynov, V. Muratova, Search for $5.5 \mathrm{MeV}$ solar axions produced in p(d, $\left.{ }^{3} \mathrm{He}\right) \mathrm{A}$ reaction," Bull. Rus. Acad. Sci. Phys. 74, 805 (2010) [arXiv:1007.3387v1 [hep-ex]].

[4] A. Derbin, A. Kauynov, V. Muratova, D. Semenov, E. Unzhakov, "Constraints on the axion-electron coupling for solar axions produced by Compton process and bremsstrahlung," Phys. Rev. D 83, 023505 (2011) [arXiv:1101.229 [hep-ex]]. 